\title{
TV/Series
}

12 | 2017

Littérature et séries télévisées/Literature and TV series

\section{Livre et pouvoir des mots dans les séries de science-fiction}

Florent Favard

\section{(2) OpenEdition \\ Journals}

Édition électronique

URL : http://journals.openedition.org/tvseries/2136

DOI : 10.4000/tvseries.2136

ISSN : 2266-0909

Éditeur

GRIC - Groupe de recherche Identités et Cultures

Référence électronique

Florent Favard, «Livre et pouvoir des mots dans les séries de science-fiction », TV/Series [En ligne],

12 | 2017, mis en ligne le 20 septembre 2017, consulté le 21 avril 2019. URL : http://

journals.openedition.org/tvseries/2136; DOI : 10.4000/tvseries.2136

Ce document a été généré automatiquement le 21 avril 2019

\section{(c) (1)}

TV/Series est mis à disposition selon les termes de la licence Creative Commons Attribution - Pas d'Utilisation Commerciale - Pas de Modification 4.0 International. 


\title{
Livre et pouvoir des mots dans les séries de science-fiction
}

\author{
Florent Favard
}

1 Dans son poème intitulé «The Speed of Darkness », Muriel Rukeyser disait: "/ The Universe is made of stories, / not of atoms ${ }^{1}$. »

2 La plupart des articles de ce numéro se concentrent sur des adaptations télévisuelles d'œuvres littéraires, ou sur la représentation de la littérature à l'écran; c'est dans cette seconde veine que s'inscrit le présent travail, avec toutefois une insistance sur la matérialité de l'objet livre en tant que tel, qu'il soit support de fictions, de textes religieux ou historiques. Il s'agira ici de s'intéresser aux séries narrativement complexes ${ }^{2}$ contemporaines qui déploient sur le long terme une intrigue macroscopique; le sousensemble visé concerne des séries feuilletonnantes faisant la promesse d'un dénouement, c'est-à-dire structurant cette intrigue macroscopique de façon téléologique, toute entière portée vers une conclusion qui, dans le modèle de production états-unien, imprévisible et fortement concurrentiel, est presque impossible à mettre en place ${ }^{3}$.

3 Les trois programmes qui composent le présent corpus sont parvenus au dénouement promis des années plus tôt. Qu'il s'agisse de la quête de la Terre dans Battlestar Galactica (Sci-Fi, 2003-2009), de la Guerre des Ombres dans Babylon 5 (PTEN>TNT, 1993-1998) ou de l'énigme en temps non-linéaire proposée par l'ère du Onzième Docteur dans Doctor Who (BBC, 1963-1989; 2005-présent), ces séries font la promesse d'un dénouement et se structurent tout entières autour d'une intrigue macroscopique téléologique. Elles possèdent aussi la particularité - et c'est là l'objet de cet article - d'articuler tout ou partie de cette intrigue autour d'ouvrages présents à l'intérieur du monde fictionnel, et ayant une grande influence sur les décisions des personnages. Au travers de l'analyse successive de ces trois séries et de leur rapport à l'objet livre, il s'agira d'explorer la façon dont ces ouvrages peuvent introduire une tension d'ordre générique, renvoyer à la légitimité de la forme romanesque ou encore proposer un discours réflexif sur l'écriture. 


\section{Bible des étoiles, bible des scénaristes : Battlestar Galactica}

Battlestar Galactica structure son intrigue macroscopique autour des Rouleaux Sacrés, un texte saint qui permet de relancer le récit. La série débute par l'annihilation des Douze Colonies, perpétrée par les Cylons, des intelligences artificielles créées par les Colons euxmêmes. Les survivants de cette attaque se réfugient à bord d'une flotte spatiale hétéroclite guidée par le dernier vaisseau de guerre encore en fonction - le Battlestar Galactica éponyme. Harcelée par les vaisseaux mères Cylons, la Flotte est aussi infiltrée par des modèles cylons à apparence humaine, et c'est alors, peu à peu, que se dessine une course-poursuite paranoïaque à travers la galaxie. Colons et Cylons cherchent la Terre, la mythique Treizième Colonie, un objectif annoncé dès la minisérie qui sert d'épisode pilote au programme. Cet objectif structure l'intrigue macroscopique de la série, en ce qu'il est au cœur de sa promesse de dénouement et rythme chaque saison à mesure que la Flotte progresse (difficilement) dans sa quête, perd espoir, se désagrège, change jusqu'à ne plus se reconnaître - pour le meilleur et pour le pire.

5 C'est dans la minisérie, lors de l'office religieux rendu aux victimes, devant un public désemparé, qu'Adama interpelle la prêtresse Elosha:

ADAMA. [...] Life here began out there. Those are the first words of the Sacred Scrolls, and they were told to us by the Lords of Kobol, many countless centuries ago. And they made it perfectly clear that we are not alone in this universe. Elosha, there's a thirteenth colony of humankind, is there not?

ELOSHA. Yes. The Scrolls tell us a thirteenth tribe left Kobol in the early days. They travelled far and made their home upon a planet called Earth, which circled a distant and unknown star.

ADAMA. It's not unknown. I know where it is!

6 Peu après, Roslin explique à Adama qu'elle sait qu'il a raconté un mensonge, et qu'aucun Colon n'a jamais connu l'emplacement de la Terre, légende perdue au sein des « Rouleaux Sacrés ", le livre saint de la religion polythéiste des Douze Colonies ${ }^{4}$. Avec une certaine ironie, c'est pourtant la présidente elle-même qui, après le diagnostic de son cancer, va se retrouver comparée au "leader mourant» annoncé par la prophétie de la Pythie contenue dans les Rouleaux Sacrés ; c'est ainsi qu'Elosha apparaît plusieurs fois durant la saison 1, dès The Hand of God (1.10), et avec elle, les Rouleaux Sacrés, sous forme de rouleaux ou d'ouvrages reliés dont seules les pointes taillées en biseau les distinguent des livres que nous connaissons. Lorsque les Rouleaux Sacrés ne sont pas présents, c'est Elosha qui les personnifie, récitant leurs versets.

On notera que la fonction d'Elosha, jusqu'à sa mort en début de saison 2, est équivalente à celle des Rouleaux Sacrés que Roslin récupère ensuite sur son corps sans vie : relancer l'intrigue macroscopique de la série. Il n'est guère étonnant qu'Elosha et le Livre de la Pythie fassent leurs principales apparitions en fin de saison 1, ainsi qu'en début de saison 2 et 3 , des moments critiques à l'échelle du programme, durant lesquels la narration atteint un tournant. L'exploration de la planète Kobol, justifiée par la Pythie, génère la monté des enjeux qui clôt la saison 1 ; cet arc narratif ne se conclut qu'en début de saison 2 (2.5-2.6) pour lancer la Flotte vers la prochaine étape. L'établissement sur New Caprica, planète que les Colons pensent adéquate pour leur survie, et la guérison (temporaire) de Roslin viennent contredire le texte prophétique en fin de saison 2 : impasse pour les 
Colons, occasion d'un basculement majeur pour l'intrigue macroscopique de la série, la fuite de New Caprica envahie par les Cylons permet à la saison 3 de revenir sur le « droit chemin» dicté par la Pythie, replaçant à l'ordre du jour l'objectif qui constitue la promesse de dénouement de Battlestar Galactica.

D'un point de vue scénaristique, ce livre constitue un cliché que le site TvTropes ${ }^{5}$ répertorie sous le nom de "Tome of Prophecy ${ }^{6} »$ : un ouvrage qui impose un certain déterminisme à la progression tout en possédant une marge d'erreur (ici, via l'interprétation des métaphores). À raison, TvTropes catalogue les "Tomes of Prophecy " comme des MacGuffin - au sens hitchcockien - c'est-à-dire des éléments qui font avancer l'intrigue de façon quelque peu artificielle, sans avoir d'autre fonction explicite: quand bien même le Livre de la Pythie porte aussi sur ses épaules une partie de la charge thématique et symbolique de la série, il est d'abord un moteur de l'intrigue. De façon bien pratique pour l'écriture progressive que nécessite une série télévisée au long cours, les personnages ne cherchent jamais à lire toutes les étapes décrites par le livre d'un coup : ainsi le Livre de la Pythie peut-il suggérer que "tout est déjà écrit » (dans le monde fictionnel comme dans la salle d'écriture des scénaristes) tout en esquivant de possibles impasses narratives, laissant les scénaristes libres de rediriger le récit si besoin. Le Livre de la Pythie, appréhendé de façon fragmentaire, contribue ainsi à la relance périodique de la narration, respectant en cela l'équilibre entre "satisfactions temporaires" et « renouvellement de l'intrigue » que doivent maintenir les long-term television narratives au sens de David Lavery?.

La série propose ainsi plusieurs séances d'exégèse lors desquelles les personnages, tels des moines, se rassemblent autour du Livre de la Pythie pour le déchiffrer, explorer la subtilité de ses métaphores, et révéler la prochaine étape qui les guidera vers la Terre. Écrit à la main, illustré, le Livre de la Pythie (la seule section des Rouleaux Sacrés qui soit dévoilée en profondeur par la série) a ce souffle authentique qui évoque le carnet de voyage ; il raconte, d'ailleurs, un voyage, celui-là même qu'entreprennent les Colons en quête de la planète bleue. Lorsque, dans la saison 1, il guide l'humanité vers la planète Kobol, le livre saint affirme à la fois sa valeur de vérité et sa dimension mystique lorsqu'il valide les visions et les convictions de Laura Roslin.

10 Dans Kobol Last Gleaming, Part 1 (1.12), la présidente et son équipe consultent les relevés astrométriques de la planète Kobol; elle aperçoit sur les clichés une cité, là où Elosha et son assistant Billy voient des ruines. Elle tente de décrire sa vision ; Elosha ouvre le Livre de la Pythie pour dévoiler une gravure représentant l'antique cité aujourd'hui détruite. Si les relevés sont authentiques, c'est pourtant le livre saint qui dévoile la véritable nature de Kobol, planète d'où seraient issues les Douze Colonies. Les relevés comme le livre sont filmés sans réelle théâtralisation, mais le support moderne, sur lequel Roslin projette son hallucination (montrée à l'écran mais invisible aux autres personnages) échoue à représenter ce que révèle l'antique volume. Roslin a un message divin à transmettre à la Flotte mais, invariablement, c'est le Livre de la Pythie qui le valide.

11 Ces scènes d'exégèse sont toujours l'occasion de mettre au même niveau d'exactitude, et d'authenticité, le texte religieux et les relevés astrométriques: la plus marquante intervient dans Torn (3.6), lorsque le sous-lieutenant Gaeta reprend les travaux du docteur Baltar, et cherche la prochaine étape du voyage de la Flotte en combinant le Livre de la Pythie sous une forme archaïque (des rouleaux manuscrits) et une carte de la galaxie. Les métaphores du texte deviennent alors des repères célestes: 
GAETA. Look at this passage. "And the caravan of the heavens was watched over by a great lion with a mighty blinking eye, red and blue." Exactly.

ADAMA. Exactly what? You're looking for a lion's head?

ROSLIN. With a mighty blinking eye.

ADAMA. Blinking.

ROSLIN. Well, they're scrolls. They speak in metaphors.

GAETA. Initially, I thought the doctor might be off his meds as well, sir. But then I found this note, here, where he had written "blinking equals pulsar".

ROSLIN. That means a star, right?

GAETA. Plural, actually. They're the rotating cores of dead stars. They emit a blast of radio waves. From a distance, they appear to...

ROSLIN. Blink.

GAETA. Right. The doctor found two in very close mutual orbit within this sector. The spectrographic readings that I found show one will appear to be red, and one will appear to be blue. Now these pulsars appear to be in this nebula. We have never had a direct look at this area. But it is possible with a couple of eyeballs out there, they might look at the nebula and see...

ADAMA. A giant lion's head ${ }^{8}$.

12 Le vocabulaire scientifique, verbal et visuel, associé à la science-fiction, côtoie ainsi les textes anciens et les prophéties plus communes à la fantasy, soutenant le discours sur la religion et le divin porté par la série. Ce livre saint cristallise la vision cyclique du temps adoptée par les Colons et les Cylons, les deux camps répétant à l'envie que « all of this has happened before, and all of this will happen again. " Les Douze Colonies ont elles-mêmes été créées à la suite d'un autre exode, et le monde fictionnel de la série se révèle bientôt bâti sur d'autres exils, transits à travers l'espace, orchestrés en coulisses par des entités présentées comme divines. Non seulement "tout ceci est déjà arrivé », mais tout ceci a déjà été écrit.

Le Livre de la Pythie sous-tend ainsi tout un discours religieux : la série est largement influencée par le christianisme, le protestantisme, voire le mormonisme ${ }^{9}$, et ce livre saint évoque bien entendu la Bible. Questionner le livre, c'est questionner l'existence du divin, une question qui court tout le long de la série. Ainsi non seulement le Livre de la Pythie sert-il à alimenter la quête de la Terre, qui est au cœur de l'intrigue, mais il établit aussi une forte tension générique entre l'univers science-fictionnel, peuplé de vaisseaux spatiaux et d'intelligences artificielles, et le livre, support millénaire qui donne accès à une parole divine authentique. Toute prophétie qu'il soit, le Livre de la Pythie n'en acquiert pas moins valeur de vérité, puisqu'il évoque une histoire qui se répète millénaire après millénaire : il incarne cette idée de variation sur le même thème, faisant ainsi écho à la série originale Battlestar Galactica (ABC, 1978-1979), dont la création de Ronald D. Moore se veut la « réimagination ${ }^{10}$ ".

14 La saison 4, dont les scénaristes savaient qu'elle serait la dernière, possède à ce titre la scène la plus réflexive autour du Livre de la Pythie. Les Colons y découvrent la « Terre »du moins celle annoncée par la Pythie - mais il s'agit d'un champ de ruines radioactif. La seconde moitié de la saison 4 est ainsi consacrée au désespoir de la Flotte et aux conséquences de cette terrible nouvelle. Lorsque l'amiral Adama tente de convaincre la présidente Roslin de s'adresser à la Flotte dans Sometimes a Great Notion (4.11), il la trouve en train de brûler les pages du Livre de la Pythie, tandis qu'elle les tourne dans le sens contraire à la lecture.

ADAMA. What are you doing? You just gonna lie down and quit? You're the one who made me believe in this. 
Rosuin. You shouldn't have listened to me. When the Cylons first attacked, you should've held your ground and kept fighting. Because I was wrong. I was wrong about everything. And all those people who listened, and they trusted me, and they followed me, all those people - they're dead ${ }^{11}$. Colons, qui y voient à présent, au mieux, une farce cosmique. Mais ce texte religieux, cette «bible» qui soutenait l'intrigue de la série, est aussi, en un sens, la bible des scénaristes, la "note d'intention», le document qui guide l'écriture d'une série: son autodafé marque un tournant radical dans le récit après le cliffhanger de mi-saison 4 sur une Terre dévastée, cliffhanger aménagé en hâte après l'éclatement de la grève des scénaristes de 2007-2008 ${ }^{12}$. La série semble ainsi dire que «les jeux sont faits, rien ne va plus ", et orchestre de façon réflexive le démantèlement de l'intrigue macroscopique de la série, dernier " leap of faith " pour les Colons, et pour le public qui voit la série subvertir sa promesse de dénouement pour mieux la ramener sur le devant de la scène. arbitre, il n'en est rien : les Colons ne suivent plus le script, et dans le même temps va s'amorcer la section la plus chargée en allusions religieuses, lorsque dans les derniers épisodes, l'existence d'une entité supérieure (divine ou non) se trouve confirmée au sein du monde fictionnel. Il y a là une double approche basée sur la foi, une démarche " métanarrative » ("metastorytelling ») que Jason Mittell relève dans le cas de $\operatorname{Lost}^{13}$ : la foi des fans en la capacité des scénaristes à dénouer l'intrigue; la foi comme thème prédominant, les deux aspects résonnant l'un avec l'autre.

\section{Livres saints, Histoire sacrée : Babylon 5}

On retrouve dans Babylon 5 un ouvrage important, au caractère sacré, que la série emploie toutefois d'une façon différente de Battlestar Galactica.

18 La série créée par J. M. Straczynski se déroule au $23^{\text {e }}$ siècle et raconte l'histoire d'une station diplomatique prise en étau au milieu d'une guerre interstellaire. Elle met en avant un large casting de personnages, humains et extraterrestres, et parmi ces derniers, l'ambassadeur des Narns, G'Kar, qui est l'un des premiers à voir monter la menace représentée par les Ombres, une civilisation millénaire vouée à décimer les peuples de la galaxie.

Il faut noter ici que Babylon 5 est moins intéressée par les prophéties qu'elle ne l'est par l'Histoire : la mémoire du passé constitue le cœur de l'intrigue et du tissu thématique de l'ensemble de la série ${ }^{14}$, puisque cette dernière dresse les chroniques d'une guerre qui appartient déjà au passé.

20 À ce titre, le Livre de G'Quan ${ }^{15}$, un texte sacré chez les Narns, révèle les détails d'une précédente guerre menée contre les Ombres, 1000 ans plus tôt. Là encore, la dimension cyclique (celle du temps mais surtout de l'Histoire) est mise en avant : ainsi l'ouvrage renferme-t-il des informations cruciales sur la guerre contre les Ombres.

21 Les livres des Narns possèdent des caractéristiques particulières : ils sont obligatoirement manuscrits, recopiés à la main, à l'identique, et même le plus petit défaut se retrouve reporté pour conserver « intact » l'ouvrage au fil des siècles. Ils doivent être lus dans la langue d'origine, ce que conseille l'ambassadeur G'Kar au chef de la sécurité de la station Babylon 5, Michael Garibaldi. Si le Livre de G'Quan véhicule principalement la dimension 
religieuse du personnage de G'Kar (il le consulte régulièrement), l'ouvrage occupe une place prépondérante en saison 3 lorsque Garibaldi tente de le déchiffrer. Le chef de la sécurité y découvre alors un indice capital dont il fait part à l'équipe dans Ship of Tears (3.14), amenant dans le quartier général dernier cri l'ouvrage antique :

GARIBALDI. I've got it. I've got it, I've got it. It's right here. It's right - right here. [il tape le livre]

G'KAR. Do not thump the Book of G'Quan. It is disrespectful.

$[\ldots]$

GARIBALDI. A thousand years ago, ambassador. You said the last time the Shadows were on the move was a thousand years ago. G'Kar, it says here that your world was used as a base by the Ancient Enemy during that time.

G'KAR. Yes, that is correct..

GARIBALDI. All right. Read this. Right here.

G'KAR. "And the spirit of darkness moved upon the land. It screamed in the dreams of the mindwalkers. And they fell, destroyed by it, to their children and their children's children. Then did the darkness come to Narn until it was driven out by G'Quan and the last of the surviving mindwalkers."

SHERIDAN. Mindwalkers. Telepaths? They're afraid of telepaths! ${ }^{16}$ ?

On retrouve ici un schéma similaire à celui de Battlestar Galactica, avec ces séances d'exégèse de textes anciens, aux allures mystiques, au sein d'un univers assimilé au space opera. Une tension générique entre science-fiction et fantasy que Babylon 5 cultive par ailleurs, puisqu'elle accumule de nombreuses références au Seigneur des Anneaux au fil de ses cinq saisons. Cependant, plutôt que d'insister sur le religieux (un thème que la série tend à éviter ou subvertir), Babylon 5 emploie le livre comme un témoin de l'Histoire, témoin qui n'est toutefois pas inaltérable, et qui mérite toujours d'être questionné.

Si les livres des Narns sont reproduits fidèlement, plus généralement dans la série le livre est d'abord le support du mythe, de l'Histoire déformée par les générations. Cette tension est particulièrement prégnante dans l'épisode final de la saison 4, The Deconstruction of Falling Stars. Via des extraits vidéos compilés dans des archives, l'épisode dévoile le futur du monde fictionnel, 100, 500 puis 1000 ans après le présent du récit. Dans le futur le plus éloigné, la Terre a été ravagée par une guerre civile, ramenée à un niveau de technologie médiéval, mais la mémoire des événements racontés dans la série a été conservée, transcendée par le mythe, devenu une légende dont certains doutent même de la véracité. Cette légende est consignée... dans un livre. Deux moines s'interrogent sur le mythe qu'il véhicule, feuilletant des pages manuscrites, enluminées :

FRÈre MICHAEL. A fable, specifically created to match scripture, that's what the others say. And there is not one shred of evidence that [it] even existed.

FRÈRE ALWYN. There is proof. Out there, in space.

FRÈRE MICHAEL. Yes, but space is closed to us now. Just look at us, Brother Alwyn. And look at what Earth has become. Our cities are little more than villages. All those great secrets of our ancestors are almost gone.

FRÈRE ALWYN. Almost. That's why we're here. To find the ancient wisdoms and preserve them. Why, we've already found and restored so many books.

FRÈRE MICHAEL. Yes, but we are not one inch closer to those flying machines that the books talk about, or to the stars. And if the truth really does lie in the stars then how will we ever know the truth? Look, the others. [il feuillette le livre] The blessed Sheridan who lived and died and returned from the dead and was taken bodily into heaven. And lvanova the strong and Delenn the wise. They could all be fables for all we know ${ }^{17}$. 
même que Frère Michael doute, le public qui a suivi la série sait que les personnages mentionnés ont vraiment existé ; l'annonce de la mort de Sheridan est même, à ce moment du récit, proleptique, puisqu'elle n'intervient qu'à la fin de la série. La défamiliarisation ainsi produite permet de questionner la pertinence du livre, mais souligne aussi la nécessité d'un devoir de mémoire éclairé, l'obsession thématique de la série. Dans ce passage de l'épisode, ce sont des vidéos de surveillance dans le monastère qui nous permettent de voir les moines au travail. Ainsi la vidéo apparaît comme véhicule d'une certaine authenticité, tandis que le livre déforme. Sauf que l'extrait est à replacer dans un épisode qui s'intéresse non pas à la distinction entre Histoire authentique et mythe mensonger, mais entre deux perceptions de l'Histoire : celle qui met l'accent sur des forces de l'Histoire, et celle qui met l'accent sur l'individu, ici transformé en figure mythique, pour le meilleur et pour le pire. Dans Babylon 5, c'est le livre qui forge les héros et héroïnes, et il appartient aux personnages de distinguer le vrai du faux, le fait réel de sa mythification.

La même problématique structure le personnage de l'ambassadeur G'Kar, au travers d'un autre livre: le sien. Conçu après le conflit entre son peuple, les Narns, et leurs oppresseurs, les Centauris, le Livre de G'Kar fait des apparitions récurrentes durant les saisons 3 à 5 : il relate son combat et se présente comme un manifeste de politique mêlé de philosophie. Mais publié sans son consentement durant la saison 5, le manuscrit l'érige, malgré lui, en figure religieuse, égale au divin G'Quan (The Ragged Edge, 5.12). G'Kar devient alors maître à penser, et témoin privilégié du conflit entre les Narns et les Centauris. Mais tandis qu'il cherche à enseigner la prise de recul et l'exploration éclairée de l'Histoire, les Narns se limitent à son livre, qui est pour eux aussi inaltérable que celui de G'Quan - l'ouvrage a d'ailleurs été recopié à l'identique... avec une tache de café laissée par Garibaldi. The Ragged Edge (5.12) donne l'illustration de ce problème lors d'une séance de lecture de G'Kar face à ses fidèles, alors que l'ambassadeur, accompagné de son garde du corps Ta'Lon, encourage à l'apaisement :

FIDÈLE. I understand what you are saying but in the book of G'Kar it says that the Centauri cannot be trusted.

G'KAR. When was that written in the book?

FIDÈLE. In the beginning.

G'KAR. Exactly. Over time, I learned, as you will learn.

FIDÈLE. The book of G'Kar is holy. If it was written under the direct inspiration of the universe as everyone believes then the whole of it must be true. How then can you go against it?

G'KAR. Show me the passage in the book. Good. Good, now put your face in the book. [Le fidèle hésite] Well, if the book is holy, and I am holy then I must help you become closer to the thoughts of the universe. Put your face in the book. The first thing we are all taught is that while outsiders cannot be trusted we can always trust a fellow Narn, yes? This is your point, is it not? Good. Then put your face in the book. [...]

[Le fidèle met son visage dans le livre]

[G'Kar le referme violemment sur lui]

TA'LON. There's lesson number one ${ }^{18}$.

Si cet extrait explique qu'il ne faut jamais accorder une confiance aveugle au livre, ou à l'auteur, la série articule par le biais des livres un discours plus large sur la nécessité d'un devoir de mémoire éclairé. Il est ici intéressant de replacer l'emploi du livre par Babylon 5 dans le contexte de production et de réception du programme. J. M. Straczynski, créateur et principal scénariste de la série (il a signé 90 de ses 110 épisodes), s’impose lui-même 
comme figure auctoriale, invitant son public à lire la série sur le mode de l'épique brechtien ${ }^{19}$ : il cherche à proposer une image du monde qui appelle à la réflexion, et n'a jamais caché par ailleurs la charge politique de son récit comme de son activité de scénariste ${ }^{20}$. Ainsi, l'exégèse menée par les personnages face au livre reflète l'attention qu'exige Straczynski de son public face à ce qu'il ne cesse d'appeler un «roman pour la télévision ${ }^{21} »$. Ce faisant, il encadre (parfois malgré lui) la série dans un discours légitimant en l'associant à une forme artistique autrement plus reconnue, celle du roman, en même temps qu'il s'impose en héritier de la science-fiction littéraire ${ }^{22}$.

Il est aussi important de replacer cette comparaison au roman - notable dans les discours sur la quality TV dans les années 1990 et $2000^{23}$ - dans le contexte spécifique des séries à promesse de dénouement, où elle résonne de façon toute particulière : elle évoque ainsi la causalité régressive genettienne ${ }^{24}$, l'idée que l'auteur d'un roman l'écrit «à rebours » en partant de la fin, un processus virtuellement impossible à mettre en place à la télévision. Il s'agit, là encore, d'une comparaison qui se veut légitimante, et que les showrunners de la série Lost (ABC, 2004-2010), Carlton Cuse et Damon Lindelof, ont aussi largement utilisée ${ }^{25}$. Mais nul autre que Straczynski n'a autant mobilisé cette idée, lui qui n'a cessé de déclarer (y compris durant la production) que les cinq saisons de la série étaient planifiées de longue date. Un pari qu'il a gagné, mais non sans mal, le départ d'un acteur principal (Michael O'Hare en fin de saison 1), et la chute du réseau PTEN qui diffusait alors la série (fin de saison 4), l'obligeant notamment à revoir sa copie. De la même façon que Battlestar Galactica, l'omniprésence du livre renvoie en miroir à cette promesse de dénouement, à une écriture qui passerait outre sa fondamentale "économie prospective ${ }^{26}$ ", son caractère progressif ${ }^{27}$, pour se rapprocher de la forme close, écrite en un bloc, qui peut évoquer le roman. Mais dans Battlestar Galactica comme dans Babylon 5, cette analogie reste indirecte, soit parce que la prophétie de la Pythie est sujette à interprétation(s), soit parce que l'analogie est dissimulée derrière une thématique portant sur le devoir de mémoire. L'emploi réflexif du livre comme métaphore d'une écriture sérielle téléologique n'est jamais plus prégnant que dans Doctor Who.

\section{Mise en récit, mise en abyme : Doctor Who}

Si dans Battlestar Galactica et Babylon 5, les livres véhiculent la dimension cyclique du temps et de l'Histoire, c'est sa non-linéarité et la réflexivité amenée par le questionnement de l'écriture qu'exploite Doctor Who. Là où les Colons sont des lecteurs et des lectrices, et l'ambassadeur G'Kar un lecteur devenu écrivain, les personnages de Doctor Who s'assument comme auteurs de leur biographie. Extraterrestre à apparence humaine voyageant à travers l'espace et le temps, souvent accompagné de terriens, le personnage éponyme est confronté à ces problématiques durant l'ère du Onzième Docteur, incarné par Matt Smith ${ }^{28}$.

Durant la cinquième saison de la série ${ }^{29}$, le Docteur et Amy Pond sont manipulés par les ennemis jurés de l'extraterrestre: ils ont puisé dans les connaissances de la jeune terrienne pour construire une légende qui paraîtrait familière au duo et le pousserait à enquêter. D'un point de vue scénaristique, la tactique prend tout son sens au regard de la série, qui, depuis son retour sur les écrans en 2005, possède une intrigue étendue sur chaque saison, et dont le dénouement constitue le season finale. Au cours de cette cinquième saison, le mystère de la « Pandorica » mentionné à de nombreuses reprises est 
à la fois un piège pour le Docteur, et pour le public qui ne s'attend pas à ce que cette « norme intrinsèque ${ }^{30}$ » de la série se retourne contre ses stratégies de lectures.

Ainsi, au $\mathrm{II}^{\mathrm{e}}$ siècle après JC, les ennemis du Docteur ont construit une fausse armée romaine et une mystérieuse prison, la Pandorica, en se basant sur des livres d'Histoires et sur le mythe de la Boîte de Pandore, dont ils ont trouvé des exemplaires chez Amy. L'épisode The Big Bang (5.13) contient ainsi une séquence d'une inquiétante étrangeté, à la frontière entre montage parallèle et alterné. Lorsque l'archéologue River Song, un autre personnage central, revient dans le présent, elle découvre chez Amy les livres d'images et les contes dont se sont inspirés les ennemis du Docteur pour construire leur piège, alors même que le montage dévoile simultanément le piège lui-même, la fiction que le Docteur et Amy croient réelle. La diégèse semble alors, un temps, se scinder en deux niveaux de narration distincts; un trouble narratif manifesté plus tard par le Docteur, qui faisant face à la mort, déclare : "We're all stories in the end ». La légende de la Pandorica, que le Docteur qualifie même de "conte de fées" dans Flesh and Stone (5.5), est en réalité authentique (elle existe), en même temps qu'elle est une fiction fabriquée de toute pièce, explicitement assimilée à un «sombre conte de fée » dans la lignée du ton de l'ère du Onzième Docteur ${ }^{31}$.

31 Mais ce n'est pas là l'usage le plus remarquable de l'objet livre par la série. Au diapason de Battlestar Galactica et Babylon 5, Doctor Who possède aussi son "Tome of Prophecy", principalement aperçu durant l'ère du Onzième Docteur (2010-2013). Il s'agit du journal de l'archéologue River Song, qui entretient une relation romantique non-linéaire avec le Docteur. Puisqu'elle voyage aussi dans le temps, mais pas forcément avec lui, elle et le Docteur ne sont pas synchrones : le journal les aide à se repérer dans le temps pour savoir "quand» chacun se situe lors de leurs nombreuses rencontres. Le journal est donc potentiellement source de spoilers, à la fois pour les personnages et le public. Des révélations que la malicieuse River Song s'amuse à distiller, anticipant ainsi sur la suite des aventures du Docteur - et sur le futur du récit. On pourrait y voir une tension générique d'ordre réflexif, puisque les spoilers, dynamisés par la «culture de la convergence » chère à Henry Jenkins ${ }^{32}$, sont ici véhiculés par un support « archaïque » (le livre papier) au regard de leur mode de transmission principalement numérique (réseaux sociaux, forums, sites de fans...). Mais il s'agit surtout d'anticiper sur la progression de l'intrigue macroscopique pour mieux véhiculer des fausses pistes à même de duper les « fans médecins-légistes ${ }^{33}$ » qui analysent le récit au peigne fin.

Le journal apparait pour la première fois en même temps que River. Dans le diptyque Silence in the Library/Forest of the Dead (4.8 et 4.9), un épisode qui se déroule dans une bibliothèque planétaire, le Docteur la rencontre pour la première fois. Mais du point de vue de River, qui a déjà vécu toute une vie avec lui, c'est la dernière fois qu'elle le croise, puisqu'elle meurt à la fin de cet épisode, se sacrifiant pour sauver la bibliothèque. Lorsque le Docteur tente de s'interposer, elle le lui interdit, car cela modifierait la ligne temporelle - et donc, tout ce qu'elle a pu vivre avec lui.

LE DOCTEUR. You can let me do this.

RIVER. If you die here, it'll mean I've never met you.

LE DOCTEUR. Time can be rewritten.

RIVER. Not those times. Not one line. Don't you dare. It's okay. It's okay. It's not over for you. You'll see me again. You've got all of that to come ${ }^{34}$. 

autrice de sa biographie, River refuse une réécriture, conservant ainsi le contrôle sur sa vie.

Silence in the Library (4.8) est l'occasion d'introduire un moment qui deviendra récurrent par la suite : la «synchronisation des biographies» entre River et le Docteur, avec le journal comme invariant narratif, véhicule du futur de l'intrigue. Lors de leur première rencontre, River remonte le fil de son journal en interrogeant celui qui ne la connait pas encore, et en mentionnant des événements futurs.

RIVER. You're doing a very good job, acting like you don't know me. I'm assuming

there's a reason.

LE DOCTEUR. A fairly good one, actually.

RIVER. Okay, shall we do diaries, then? Where are we this time? Er, going by your

face, I'd say it's early days for you, yeah? So, er, crash of the Byzantium. Have we done that yet?

L'action de « faire le journal » (« let's do diaries », dit River) est établie comme une routine - ce qui est confirmé à l'échelle de la série. River mentionne par exemple dans Silence in the Library le crash du Byzantium, un événement exploré plus tard dans le diptyque The Time of Angels/Flesh and Stone (5.4, 5.5), durant lequel les personnages vivent le crash du vaisseau nommé Byzantium. Le journal de River Song fonctionne ainsi, non pas forcément comme un réservoir de prolepses effectives (les scénaristes ne savent pas forcément s'ils montreront ces événements dans la suite de la série), mais comme un réservoir des possibles qui mettent en abyme l'écriture des séries déployant une intrigue macroscopique, une écriture progressive qui doit toujours éviter de s'engager dans des impasses narratives mais cherche aussi à donner l'impression qu'elle poursuit un objectif, qu'elle est " déjà écrite ». Là où le Livre de la Pythie évoque la bible des scénaristes dans Battlestar Galactica, le journal de River Song évoque plus précisément les scripts à venir, scripts d'ailleurs contrôlés par la seule instance auctoriale qu'est le head writer (scénariste en chef) Steven Moffat, celui-ci ayant écrit tous les épisodes mettant en scène l'archéologue du futur.

L'ère du Onzième Docteur, entre 2010 et 2013, sous la plume de Moffat, met en abyme l'activité d'écriture de façon fractale : ainsi The Angels Take Manhattan (7.5), un autre épisode mettant en scène River Song, subvertit la norme intrinsèque du journal intime pour le remplacer par un polar que lisent le Docteur, Amy et son mari Rory, en 2012, à Central Park. Rory est mystérieusement envoyé en 1938, où il retrouve River Song, tandis que le Docteur s'étonne d'entendre soudain parler d'eux dans le livre qu'il est en train de lire. C'est que celui-ci a été écrit après les événements de l'épisode, et le Docteur craint par-dessus tout que lui et Amy n'en lisent la fin avant de la vivre et verrouillent ce futur. Lorsqu'Amy rappelle au Docteur que le temps peut être réécrit, il lui explique qu'il s'agit de leur propre avenir et que s'ils le lisent, alors ils ne pourront plus rien changer. Même jeter un coup d'œil au sommaire est déjà un indice de trop : le livre a été écrit après qu'ils ont vécu cette aventure, et révèle dans les grandes lignes la fin inévitable et cruelle de l'épisode - spoilers! Là encore, c'est spécifiquement la forme écrite qui détient tant de pouvoir : l'avenir est écrit, sinon dans la pierre, au moins sur le papier. L'analogie est appuyée par la présence de la tombe de Rory dans le cimetière où le Docteur a garé son vaisseau spatio-temporel: après que les personnages pensent éviter leur destin en provoquant un paradoxe temporel, pensant faire mentir le livre, c'est le regard porté par Rory sur sa propre tombe qui scelle son destin. Lui et Amy sont renvoyés en 1938 par les 
ennemis du Docteur, sans espoir de secours ; le Docteur comprend alors que l'autrice du polar relatant les événements de l'épisode n'était autre qu'Amy, écrivant dans les années 1930. Bien que le Onzième Docteur ne cesse de répéter que «l'avenir peut être réécrit » (" time can be rewritten »), ce qui est écrit - spécifiquement, présenté comme tel dans le journal de River ou d'autres supports papier - est généralement déjà arrivé, impossible à modifier. Le livre gagne toujours à la fin, réaffirmant la toute puissance (supposée) du scénariste sur son " œuvre en progrès » au sens de Winckler.

Au-delà de l'idée évidente que « tout serait déjà écrit », l'emploi des livres, on l'a vu, peut servir les ambitions esthétiques d'une série, et mettre en abyme une éventuelle promesse de dénouement en la manifestant à l'intérieur du monde fictionnel: les personnages lecteurs deviennent alors des médiateurs réflexifs; les personnages auteurs questionnent le travail et les ambitions des scénaristes. Si ce n'est la possibilité d'une causalité régressive à la télévision, le livre interroge l'unité narrative de l'œuvre sérielle, sa capacité à faire sens une fois toutes les pages, tous les épisodes, tournés. Son statut réflexif est d'autant plus souligné dans les séries de science-fiction, où il participe d'une hybridité générique : le livre écrit, support millénaire, possède encore un grand pouvoir dans, et sur le futur.

\section{BIBLIOGRAPHIE}

ASKWITH Ivan, « “Do you even know where this is going?”: Lost's Viewers and Narrative Premeditation ", in Reading Lost, éd. Roberta Pearson, Londres, New York, I. B. Tauris, 2009, p. $159-180$.

воотн Paul, « Periodising Doctor Who », Science fiction film and television, 7.2, 2014, p. 195-215. CHAPMAN James, Inside the TARDIS: The Worlds of Doctor Who, Londres, New York, IB Tauris, 2013 (édition révisée).

CLARKE STUART Sarah, Literary Lost: Viewing television through the lens of literature, Londres, New York, Continuum, 2011.

ESCOLA Marc, «Le clou de Tchekhov. Retours sur le principe de causalité régressive », Fabula, 2010, accessible en ligne à l'adresse http://www.fabula.org/atelier.php?

Principe_de_causalite_regressive, consulté le 20 septembre 2017.

FAVARD Florent, La Promesse d'un dénouement : Énigmes, quêtes et voyages dans le temps dans les séries télévisées de science-fiction contemporaines, thèse de doctorat, dir. Pierre Beylot, Bordeaux Montaigne, 2015.

GARCIA Tristan, «Les Séries qui nous quittent, les séries que nous quittons », TV/Series, $n^{\circ} 7$, juin 2015, p. 103-114.

GENETTE Gérard, Discours du récit, Paris, Points Seuil, 1972, 1983 (édition remaniée 2007).

JAMES Edward, MENDLESOHN Farah (éds.), The Parliament of Dreams: Conferring on Babylon 5, Reading, Science Fiction Foundation, 1998. 
JENKINS Henry, Convergence Culture: Where Old and New Media Collide, Londres, New York, NYU Press, 2006 (édition révisée, 2008).

JOHNSON LEWIS Erika, « Torture, Terrorism, and Other Aspects of Human Nature », in Tiffany

Potter et C.W. Marshall, Cylons in America: Critical studies in Battlestar Galactica, Londres, New York, Continuum, 2008.

KUPPERS Petra, "Quality Science Fiction: Babylon 5's Metatextual Universe », in Cult Television, éd. Sarah Gwellian-Jones et Roberta E. Pearson, Londres, Minneapolis, University of Minnesota Press, 2004, p. 45-59.

LANCASTER Kurt, Interacting with Babylon 5: Fan performances in a media universe, Austin, University of Texas Press, 2001.

LANE Andy, The Babylon File, Londres, Virgin, 1997.

LAVERY David, « Lost and Long-Term Television Narrative », 2009, in Third Person: Authoring and Exploring Vast Narratives, éd. Pat Harrigan et Noah Wardrip-Fruin, Cambridge, Londres, MIT Press, 2009, p. 313-322.

MITTELL Jason, Complex TV: The Poetics of Contemporary Television Storytelling, New York, Londres, New York University Press, 2015.

MITTELL Jason, «Sites of participation: Wiki fandom and the case of Lostpedia », Transformative Works and Cultures, vol. 3, 2009.

OTт Bryan L., « (Re)Framing Fear: Equipment for Living in a Post-9/11 World », in Tiffany Potter et C.W. Marshall, Cylons in America: Critical studies in Battlestar Galactica, Londres, New York, Continuum, 2008.

WINCKLER Martin, Les Miroirs de la vie, histoire des séries américaines, Paris, New York, Le Passage, 2002.

WOLFE Ivan, « Why Your Mormon Neighbour Knows More about This Show than You Do », in Battlestar Galactica and Philosophy: Mission Accomplished or Mission Frakked Up?, éd. Josef Steiff et Tristan D. Tamplin, Chicago, Open Court, coll. « Popular Culture and Philosophy », 2008, p. 303-316.

\section{NOTES}

1. The Speed of Darkness: Poems, Random House, 1968.

2. Qui notamment combinent les veines épisodiques et feuilletonnantes depuis les années 1990. Voir notamment l'ouvrage synthétique de Jason Mittell, Complex TV: The Poetics of Contemporary Television Storytelling, New York, Londres, New York University Press, 2015.

3. Je me réfère ici à la terminologie déployée dans Florent Favard, La Promesse d'un dénouement : Énigmes, quêtes et voyages dans le temps dans les séries télévisées de science-fiction contemporaines, thèse de doctorat, dir. Pierre Beylot, Bordeaux Montaigne, 2015.

4. Religion qui s'inspire du panthéon gréco-romain.

5. Qui explore, sous une forme encyclopédique oscillant entre l'analyse éclairée et le second degré assumé, les mécanismes narratifs les plus communs des fictions populaires. Le caractère collaboratif qui caractérise le site ne doit pas faire oublier la richesse heuristique de TvTropes, peut-être l'une des sources collaboratives les plus complètes au monde sur les motifs et lieux communs des fictions populaires. TVtropes explore et catalogue ce que Jason Mittell nomme, à la suite de David Bordwell, les «normes extrinsèques » du récit populaire, en réinvestissant les 
«compétences endo-narratives » (Baroni) de ses interprètes. Voir Jason Mittell, Complex TV, op. cit., p. 167 ; Raphaël Baroni, La Tension narrative, 2007, partie II.

6. Voir à l'adresse http://tvtropes.org/pmwiki/pmwiki.php/Main/TomesOfProphecyAndFate. Dernière consultation le 20/09/2017.

7. David Lavery, "Lost and Long-Term Television Narrative", in Third Person: Authoring and Exploring Vast Narratives, éd. Pat Harrigan et Noah Wardrip-Fruin, Cambridge, Londres, MIT Press, 2009, p. 315 [p. 313-322].

8. À partir de 12 min 19 .

9. Ivan Wolfe, "Why Your Mormon Neighbour Knows More about This Show than You Do ", in Battlestar Galactica and Philosophy: Mission Accomplished or Mission Frakked Up?, éd. Josef Steiff et Tristan D. Tamplin, Chicago, Open Court, coll. «Popular Culture and Philosophy», 2008, p. 303-316.

10. Le terme utilisé dans les discours promotionnels.

11. À partir de $21 \min 36$.

12. Les Colons découvrent la "Terre » dans Revelations (4.10), diffusé en juin 2008. Le reste de la saison 4 n'a été écrit qu'après la grève, et diffusé en 2009. Cette ultime saison est donc divisée en deux sections aux tons et ambiances différentes.

13. Jason Mittell, op. cit., p. 323.

14. Florent Favard, «War Without End: le devoir de mémoire dans Babylon 5 » (communication), colloque Guerres en séries, UPJV, Amiens, 11-13 juin 2014. Sur le sujet, on consultera aussi The Parliament of Dreams: Conferring on Babylon 5, éd. Edward James et Farah Mendlesohn, Reading, Science Fiction Foundation, 1998

15. Une figure sainte dont le nom évoque, de façon volontaire, celui de l'ambassadeur G'Kar.

16. À partir de $38 \min 56$.

17. À partir de $34 \mathrm{~min} 30$.

18. À partir de $36 \min 39$.

19. C'est notamment l'avis de Kurt Lancaster, dans Interacting with Babylon 5 : Fan performances in a media universe, Austin, University of Texas Press, 2001, p. 13-15.

20. Ibid., notamment p. 3.

21. Voir par exemple ses déclarations compilées dans l'ouvrage de Andy Lane, The Babylon File, Londres, Virgin, 1997, p. 26.

22. Petra Kuppers, "Quality Science Fiction: Babylon 5's Metatextual Universe », in Cult Television, éd. Sarah Gwellian-Jones et Roberta Pearson, Londres, Minneapolis, University of Minnesota Press, 2004, p. 49 [p. 45-60].

23. Voir par exemple Charles McGrath, «The Triumph of the prime-time novel », The New York Times, 22 octobre 1995.

24. Explorée notamment dans son Discours du récit, Paris, Points Seuil, 1972. On consultera l'article critique de Marc Escola sur le sujet, «Le clou de Tchekhov. Retours sur le principe de causalité régressive", Fabula, 2010, accessible en ligne à l'adresse http://www.fabula.org/ atelier.php ?Principe_de_causalite_regressive. Dernière consultation le 23 octobre 2016.

25. Voir par exemple Ivan Askwith, « “Do you even know where this is going?”: Lost's Viewers and Narrative Premeditation", in Reading Lost, éd. Roberta Pearson, Londres, New York, I. B. Tauris, 2009, p. 159-180.

26. Marc Escola, op. cit.

27. Martin Winckler parle ainsi d' « œuvre en progrès » dans Les Miroirs de la vie, histoire des séries américaines, Paris, New York, Le Passage, 2002, p. 52-53.

28. Au seuil de la mort, le Docteur peut se régénérer en changeant d'apparence, justifiant ainsi les changements d'interprète au fil des décennies. À la suite de James Chapman par exemple, on notera que le programme peut être classé en ères de plusieurs façons (par interprète du Docteur, par équipe de production, ...). L'ère du Onzième Docteur signe aussi l'arrivée d'une nouvelle 
équipe de production et d'un nouveau scénariste en chef, Steven Moffat, qui apporte à la série une intrigue feuilletonnante à tiroirs s'étirant sur les saisons 5 à 7. James Chapman, à la suite de Tulloch et Jenkins, parle de «production signature » pour évoquer une ère définie par une équipe de production, dans Inside the TARDIS : The Worlds of Doctor Who, Londres, New York, IB Tauris, 2013 (édition révisée). Sur les nombreuses catégorisations possibles des ères de la série, on consultera aussi Paul Booth, «Periodising Doctor Who », Science fiction film and television, 7.2, 2014, p. 195-215.

29. Plus précisément, de la continuation de la série diffusée depuis 2005 et qui reprend la suite de la période dite « classique » de Doctor Who.

30. Jason Mittell, op. cit., p. 168.

31. Une expression («dark fairytale» dans sa version originale) souvent employée par le scénariste en chef Steven Moffat lors de la promotion de la série, notamment sa saison 5.

32. On consultera notamment l'éloquent chapitre sur les spoilers de la franchise Survivor, dans Convergence Culture: Where Old and New Media Collide, Londres, New York, NYU Press, 2006 (édition révisée, 2008), p. 25 et suivantes.

33. Jason Mittell, "Sites of participation: Wiki fandom and the case of Lostpedia ", in Transformative Works and Cultures, vol. 3, 2009.

34. Forest of the Dead, à partir de $34 \mathrm{~min} 46$.

\section{RÉSUMÉS}

Cet article s'intéresse à la représentation de l'objet livre dans des séries feuilletonnantes de science-fiction dont l'intrigue est construite de façon téléologique. Il s'agit d'explorer l'appui sur l'unité narrative et la mise en abyme de l'activité d'écriture que peuvent apporter les ouvrages centraux de Battlestar Galactica, Babylon 5 et Doctor Who; l'article s'intéresse aussi à la tension générique du cliché du livre prophétique dans un univers de science-fiction, ainsi qu'à la dimension légitimante de l'objet.

This paper deals with the representation of books in serialized sci-fi TV series with an ongoing plot constructed in a teleologic fashion. It explores issues of narrative unity and self-reflexive writing introduced by the books appearing in Battlestar Galactica, Babylon 5 and Doctor Who; it also looks at the legitimizing power of books, and the generic tension caused by the presence of prophetic books in a sci-fi universe.

\section{INDEX}

Keywords : books, narration, plot, reflexivity Battlestar Galactica, ending, Dr Who, Babylon 5 Mots-clés : livre, narration, intrigue, dénouement, réflexivité, Battlestar Galactica, Dr Who, Babylon 5 


\section{AUTEUR}

\section{FLORENT FAVARD}

Florent Favard est Docteur en Études Cinématographiques et Audiovisuelles à l'Université Bordeaux Montaigne. Il travaille sur la complexité narrative des séries télévisées, les genres de l'imaginaire et le transmedia storytelling, dans une perspective narratologique contextualiste et transmédiatique.

Florent Favard holds a PhD in Cinema and Audiovisual Studies at Bordeaux Montaigne University. He works on tv series narrative complexity, science fiction and fantasy genres, and transmedia storytelling, within the field of transmedial narratology. 